\title{
FUENTES DE INVESTIGACIÓN: LAS BASES DE DATOS ESPECIALIZADAS
}

EBOR FAIRLIE FRISANCHO*

E-mail: efairlie@unmsm.edu.pe

\begin{abstract}
RE S UMEN
El presente artículo busca proporcionar información a los docentes sobre las bases de datos internacionales a las que pueden acceder a través de la Universidad, que podrán servir como referencia para los artículos y proyectos de investigación que realizan. Además presentamos un avance de la situación de San Marcos respecto a otras instituciones en cuanto al uso del internet como fuente de información para investigaciones docentes.
\end{abstract}

Palabras clave: Ranking mundial de universidades, bases de datos especializadas, artículos de investigación, proyectos de investigación.

\section{ABSTRACT}

This article tries to provide enough information given by the Internet for teacher's research. Besides we are presenting the use of the Internet at San Marcos University with respect to other institutions

Keywords: University worldwide ranking, specified database. 
Si revisamos el Ranking Universitario Mundial 2004 elaborado por la Universidad de Shangai, en China, no debemos asombrarnos que la Universidad Nacional Mayor de San Marcos (UNMSM) no figure ni remotamente entre las primeras 500 universidades que presenta este ranking.

Existen muchas razones que explican esta situación, como la crisis en el nivel educativo universitario, falta de apoyo e inversión del Estado, falta de una política de desarrollo educativo, entre otros; pero uno de los factores que consideramos particularmente importantes son los proyectos de investigación que realizan los docentes en las Facultades de nuestra universidad.

En ese sentido, veamos los criterios en los que la Universidad de Shangai se basa para valorar cada una de las instituciones que son calidad de la educación, calidad de la plana docente, resultados de la investigación y dimensión de la institución.

Cada unos de estos factores incluyen un total de seis indicadores; a su vez, cada uno de ellos tiene un peso, como podemos apreciar en el siguiente cuadro:

\begin{tabular}{|c|c|c|}
\hline CRITERIOS & INDICADORES & PESO \\
\hline $\begin{array}{l}\text { Calidad de la } \\
\text { educación }\end{array}$ & $\begin{array}{l}\text { Graduados que han obtenidos } \\
\text { Premios Nóbel y/o Medallas Field }\end{array}$ & $10 \%$ \\
\hline \multirow{2}{*}{$\begin{array}{l}\text { Calidad de la } \\
\text { plana docente }\end{array}$} & $\begin{array}{c}\text { Docentes que han ganado Premios } \\
\text { Nóbel y/o Medallas Field }\end{array}$ & $20 \%$ \\
\hline & $\begin{array}{c}\text { Investigaciones citadas } \\
\text { frecuentemente en } 21 \text { categorías } \\
\text { subjetivas* }\end{array}$ & $20 \%$ \\
\hline \multirow{2}{*}{$\begin{array}{l}\text { Resultados de } \\
\text { investigación }\end{array}$} & $\begin{array}{c}\text { Artículos publicados en las revistas } \\
\text { Nature \& Science* }\end{array}$ & $20 \%$ \\
\hline & $\begin{array}{c}\text { Artículos citados en el Science } \\
\text { Citation Index expanded y el Social } \\
\text { Science Citation Index* }\end{array}$ & $20 \%$ \\
\hline $\begin{array}{l}\text { Dimensión de } \\
\text { la institución }\end{array}$ & $\begin{array}{l}\text { Performance académica con } \\
\text { respecto a la dimensión de la } \\
\text { institución }\end{array}$ & $10 \%$ \\
\hline \multicolumn{2}{|r|}{ TOTAL } & $100 \%$ \\
\hline
\end{tabular}

Fuente: Ranking Universitario Mundial 2004 elaborado por la Universidad de Shangai-China, publicado por el Rectorado de la Universidad Nacional Mayor de San Marcos en octubre de 2004.

Observamos que exactamente el $60 \%$ del peso total de los indicadores (*), corresponden a artículos y trabajos de investigación publicados o citados por revistas científicas.

De los criterios mencionados, explicaremos específicamente tres vinculados con las investigaciones o la producción de nuevos conocimientos:
- Número de investigaciones citadas frecuentemente en 21 categorías subjetivas en ciencias naturales, medicina, ciencias físicas, ingeniería y ciencias sociales durante el período 1981 a 1999. La definición exacta de estas categorías se encuentran en el sitio web del Instituto de Información Científica (www.isihighlycited.com).

- Número de artículos publicados en las revistas Nature y Science entre 1999 y el 2003. En este caso se distingue el orden de afiliación del autor en dichas revistas. Igualmente se incluyen a las instituciones especializadas en humanidades y ciencias sociales, en las cuales este puntaje no está considerado y el peso de este indicador es repartido en otros indicadores (www.isiknowledge.com).

- Número total de artículos citados en el Science Citation Index-expanded y el Social Science Citation Index del año 2003. Solamente se consideran citaciones de artículos publicados (www.isiknowledge.com).

Por ser la primera universidad pública del Perú y por la tradición que nos caracteriza, debería correspondernos dar el ejemplo, pero creemos que no se está cumpliendo el objetivo de generar conocimientos, que es el objetivo principal de toda Universidad.

Centrémonos en los artículos de investigación realizados en nuestra facultad. Según las últimas ediciones de la revista de investigación aquí producida (décima, décima primera, décima segunda y décima tercera edición), se obtienen las siguientes estadísticas:

* Docentes que escriben artículos. Observamos que desde el 2003, disminuye considerablemente el número de docentes que escriben artículos de investigación, y por lo tanto la producción científica de nuevos conocimientos. Hay que recordar que un $60 \%$ de puntaje del Ranking Mundial de Universidades hace referencia a la producción en investigación. Adicional a esto, los artículos publicados en este tipo de revistas tienen puntaje para la promoción de docentes y son muy valorados también en el ámbito internacional.

\begin{tabular}{|c|c|c|c|}
\hline Rev. N. ${ }^{\circ} 10$ & Rev. N.11 & Rev. N. 12 & Rev. N. 13 \\
\hline 16 & 12 & 14 & 10 \\
\hline
\end{tabular}


* Artículos sin ninguna referencia bibliográfica. Notamos que felizmente han bajado considerablemente a cero (0) el registro de los artículos sin alguna nota o referencia bibliográfica. Hay que recordar que en el campo de las ciencias empresariales, para deducir algún tipo de conocimiento, es necesario tener fundamentos válidos para derivar diversas conclusiones.

\begin{tabular}{|c|c|c|c|}
\hline Rev. N. $\mathbf{1 0}$ & Rev. N. 11 & Rev. N.o 12 & Rev. N. $\mathbf{1 3}$ \\
\hline 7 de 15 & 2 de 11 & 3 de 11 & 0 de 10 \\
\hline
\end{tabular}

* Docentes que hacen referencia a páginas web varias. Advertimos que los artículos hacen escasa referencia a páginas web de donde toman la información, e inclusive muchas de ellas no son de instituciones de prestigio o reconocidas a nivel mundial. Hay que prestar atención a que ningún artículo hace referencias a alguna base de datos internacional que contienen títulos y publicaciones de alto nivel científico mundial como es el PROQUEST, ABI/INFORM, BIS, HCR, entre los más importantes del medio.

\begin{tabular}{|c|c|c|c|}
\hline Rev. N. $\mathbf{1 0}$ & Rev. N. 11 & Rev. N. 12 & Rev. N. 13 \\
\hline 6 & 1 & 0 & 4 \\
\hline
\end{tabular}

En resumen, queremos demostrar que falta un mayor incentivo para proyectos de investigación, artículos de investigación y sobre todo hacer referencia a investigaciones citadas frecuentemente a nivel internacional.

\section{LAS BASES DE DATOS}

En la actualidad, la Universidad Nacional Mayor de San Marcos se encuentra suscrita a bases de datos internacionales para diversas especialidades, destacando aquellas orientadas a las ciencias básicas.

A continuación indicaremos cuáles son las bases que la Oficina General del Sistema de Bibliotecas y la Biblioteca Central brindan en forma gratuita para toda la comunidad sanmarquina:

- Proquest. Es la principal base de datos a la que la universidad se encuentra suscrita desde setiembre del año 2004 a través de la Oficina General del Sistema de Bibliotecas y Biblioteca Central. El acceso es gratuito desde el campus universitario, y requiere de una clave para alumnos o docentes que lo solici- ten para sus trabajos o investigaciones. Esta base de datos -conocida internacionalmentecontiene artículos en texto completo ( full text), resúmenes (abstracts) de reconocidas publicaciones periódicas (revistas y periódicos), del ámbito académico y científico.

Esta gran base de datos contiene a su vez 26 bases de datos de diversos temas y especialidades del conocimiento, entre los cuales mencionaremos que para la rama empresarial tenemos por ejemplo:

* ABI/INFORM Global: negocios, economía, administración, comercio internacional, marketing, entre otros (2 450 títulos).

* ABI Trade \& Industry: comercio industrial, comunicaciones, construcción, finanzas, entre otros (1 038 títulos).

* Banking Information Source: servicios financieros, industria, banca, tendencias bancarias, entre otros (438 títulos).

* Career \& Technological Education: ciencias aplicadas, tecnología informática, entre otros (484 títulos).

* Hoover's Company Records: negocios, economía, finanzas, entre otros (referencias a 15000 empresas).

* Proquest Computing: bases de datos, software, e-commerce, redes, intranet, desarrollo de tecnología, entre otros.

* Proquest Legal : derecho, derecho empresarial, derecho civil, entre otros.

* Proquest Research Library: contiene el Research Library Core en donde se puede encontrar información de negocios, humanidades, ciencias sociales, entre otros.

Esta inmensa base de datos tiene más de 6500 títulos de revistas científicas internacionales, y cuenta asimismo con más de dos millones de tesis de maestría y doctorado en todas las áreas del conocimiento.

La búsqueda es personalizada por autor, temas, publicación, tipo de revista, año, tesauro, entre otros, a través de opciones amigables tipo texto para el usuario y entendibles porque la interfase de trabajo se puede configurar para el idioma castellano. El dominio del idioma inglés a un nivel intermedio es necesario, ya que entre un 90 a 95\% de los títulos de las publicaciones se encuentran en inglés y muy pocos en español. Por último, tiene la facilidad de enviar los ar- 
tículos por correo electrónico, imprimirlos y hasta exportarlos en diferentes formatos.

- Instituto de Información Científica (Current Contents del Institute of Scientific Information-ISI) . Importante base de datos y a la que la universidad accede a través del Consejo Superior de Investigaciones. Tiene acceso on-line monousuario a los contenidos actualizados de un máximo de siete disciplinas diferentes. Adicionalmente, esta web permite la búsqueda en una colección superior de sitios web académicos evaluados y permite acceder a documentos en tres tipos de recursos: pre prints, información original y actividades de investigación.

No nos olvidemos que tres de las seis categorías que corresponden al 60\% del Ranking Universitario Mundial son tomadas por artículos de revistas y publicaciones que aparecen en esta web, más conocida como la web del conocimiento (ISI Web of knowledge) y que proporciona acceso a información bibliográfica completa de cerca de las 8000 revistas más destacadas a nivel académico y de más de 2000 libros de todo el mundo, que en la mayoría de los casos no están a nuestro alcance.

Asimismo, al igual que el PROQUEST, muestra un cuadro completo de los campos de investigación global recientes, con la combinación de la cobertura tanto de revistas como de libros en sitios web, incluyendo resúmenes de autor, direcciones del autor y más información por registro bibliográfico que en otros recursos.

- Scirus. Es la tercera base de datos que también consideramos importante. Es un buscador tipo Google, que cuenta con más de 167 millones de páginas web exclusivamente con contenido científico, filtrando aquellas que no lo tienen, revisando puntualmente artículos en archivos PDF y Postscript, que usualmente son invisibles para los demás buscadores, y lo hace de acuerdo a la relevancia de la búsqueda y por fechas.

Algo más interesante que tiene este buscador es la relevancia de la búsqueda, ya que busca una palabra a nivel de ranking estático y además tiene una estadística interna de búsqueda que rankea las páginas de acuerdo a cual de ellas tienen mayores referencias.

Adicional a las páginas web, este motor de búsqueda consulta las mejores nueve fuen- tes académicas de más alto prestigio que interesan a la rama empresarial. Podríamos tener Scitation, ScienceDirect Online, Cogprints, E-Print ArXiv, LexisNexisy el Project Euclid; aclarando que nuevamente estas publicaciones están en inglés.

Finalmente, diremos que la Universidad también está inscrita en las bases de datos scielo y Cibertesis, además de publicaciones por Internet que la Biblioteca Central ha difundido.

Teniendo una gran cantidad de artículos, libros, publicaciones, proyectos, tesis e investigaciones a los cuales se puede acceder a través de estas grandes bases de datos y motores de búsqueda por internet y que además son totalmente gratuitos, es oportuno promocionar el uso masivo de estos recursos para nuestros trabajos, investigaciones, proyectos y, por qué no, artículos de investigación, a fin de ganar valor científico y académico.

\section{BIBLIOGRAFÍA}

Gestión en el Tercer Milenio. Revista de Investigación de la Fac. de Ciencias AdministrativasUNMSM. Año 5, N.॰ 10, enero de 2003.

Gestión en el Tercer Milenio. Revista de Investigación de la Fac. de Ciencias AdministrativasUNMSM. Año 6, N.॰ 11, junio de 2003.

Gestión en el Tercer Milenio. Revista de Investigación de la Fac. de Ciencias AdministrativasUNMSM. Año 6, N. 12, diciembre de 2003.

Gestión en el Tercer Milenio. Revista de Investigación de la Fac. de Ciencias AdministrativasUNMSM. Año 7, N. ${ }^{\circ}$ 13, julio de 2004.

Rectorado de la Universidad Nacional Mayor de San Marcos. 2004. Ranking Universitario Mundial. Elaborado por la Universidad de Shangai-China. Octubre.

Instituto de Información Científica (ISI) web del conocimiento. http: \|www.isiknowledge.com y http: \|www.isihighlycited.com

Biblioteca Central de la Universidad Nacional Mayor de San Marcos. http: \|sisbib.unmsm.edu.pe

Consejo Superior de Investigaciones de la Universidad Nacional Mayor de San Marcos. http: \\csi.unmsm.edu.pe 